(c) The Authors 2015. This is an Open Access article, distributed under the terms of the Creative

Commons Attribution licence (http://creativecommons.org/licenses/by/3.0/), which permits unrestricted

re-use, distribution, and reproduction in any medium, provided the original work is properly cited.

\title{
Fatty acid profile of plasma NEFA does not reflect adipose tissue fatty acid profile
}

\author{
Celia G. Walker ${ }^{1, *}$, Lucy M. Browning ${ }^{1}$, Lynne Stecher ${ }^{2,3}$, Annette L. West ${ }^{4}$, Jackie Madden ${ }^{4}$, \\ Susan A. Jebb ${ }^{1,5}$ and Philip C. Calder ${ }^{4,6}$ \\ ${ }^{1}$ MRC Human Nutrition Research, Elsie Widdowson Laboratory, Cambridge CB1 9NL, UK \\ ${ }^{2}$ MRC Biostatistics Unit Hub for Trials Methodology Research, Institute of Public Health, University Forvie Site, Cambridge \\ CB2 OSR, UK \\ ${ }^{3}$ Else-Kröner-Fresenius Center for Nutritional Medicine, Technische Universität München, Munich D-80333, Germany \\ ${ }^{4}$ Human Development \& Health Academic Unit, Faculty of Medicine, University of Southampton, Southampton General \\ Hospital, Southampton SO16 6YD, UK \\ ${ }^{5}$ Nuffield Department of Primary Care Health Sciences, University of Oxford, Radcliffe Observatory Quarter, Oxford \\ OX2 6GG, UK \\ ${ }^{6}$ NIHR Southampton Biomedical Research Centre, University Hospital Southampton NHS Foundation Trust and University of \\ Southampton, Southampton General Hospital, Southampton SO16 6YD, UK
}

(Submitted 18 December 2014 - Final revision received 8 May 2015 - Accepted 29 May 2015 - First published online 24 July 2015)

\section{Abstract}

Adipose tissue (AT) fatty acid (FA) composition partly reflects habitual dietary intake. Circulating NEFA are mobilised from AT and might act as a minimally invasive surrogate marker of AT FA profile. Agreement between twenty-eight FA in AT and plasma NEFA was assessed using concordance coefficients in 204 male and female participants in a 12-month intervention using supplements to increase the intake of EPA and DHA. Concordance coefficients generally showed very poor agreement between AT FA and plasma NEFA at baseline SFA: 0.07; MUFA: 0.03; $n$-6 PUFA: $0 \cdot 28 ; n$-3 PUFA: 0.01). Participants were randomly divided into training (70\%) and validation (30\%) data sets, and models to predict AT and dietary FA were fitted using data from the training set, and their predictive ability was assessed using data from the validation set. AT $n$-6 PUFA and SFA were predicted from plasma NEFA with moderate accuracy (mean absolute percentage error $n$ - 6 PUFA: $11 \%$ and SFA: $8 \%$ ), but predicted values were unable to distinguish between low, medium and high FA values, with only $25 \%$ of $n-6$ PUFA and $33 \%$ of SFA predicted values correctly assigned to the appropriate tertile group. Despite an association between AT and plasma NEFA EPA $(P=0 \cdot 001)$ and DHA $(P=0.01)$ at baseline, there was no association after the intervention. To conclude, plasma NEFA are not a suitable surrogate for AT FA.

Key words: NEFA: Adipose tissue fatty acids: EHA supplementation: DHA supplementation: Prediction equations: Surrogate markers

The fatty acid (FA) composition of adipose tissue (AT) is considered to reflect habitual intake of FA, particularly of $n-3$ and n-6 PUFA ${ }^{(1)}$, trans-FA ${ }^{(2)}$ and SFA from dairy sources ${ }^{(3)}$. However, the AT content of some SFA, such as palmitic acid, does not reflect dietary intake well, probably because those FA are synthesised de novo, as well as coming from the diet ${ }^{(1,4,5)}$. AT FA composition is also regulated by other factors; for example, increased physical activity is associated with a higher proportion of SFA with a correspondingly lower proportion of MUFA $^{(6)}$, and insulin resistance is associated with an increased proportion of palmitic acid and a decreased proportion of PUFA within $\mathrm{AT}^{(7)}$. Inflammation within $\mathrm{AT}$ is implicated in the development of insulin resistance and other aspects of metabolic syndrome ${ }^{(8,9)}$, and AT inflammation is influenced by FA availability ${ }^{(10)}$. Therefore, there is much interest in knowing the FA composition of AT for understanding both dietary intake and AT biology. However, AT biopsy is an invasive procedure and is not practical to conduct in large trials, in the field or in vulnerable populations. In the fasting state, circulating NEFA are primarily derived from AT lipolysis ${ }^{(1,11)}$, and if the composition reflects that of AT, NEFA may provide a minimally invasive surrogate marker of AT FA composition. Previous studies have reported good correlations between FA species in NEFA and AT, although these studies have been limited to a comparatively

Abbreviations: AT, adipose tissue; FA, fatty acids.

* Corresponding author: C. G. Walker, fax +44 1223437 515, email Celia.Walker@mrc-hnr.cam.ac.uk 
small number of participants, to cohorts that are not representative of the population or to studying a limited number of $\mathrm{FA}^{(1,5,12)}$. In the current study, we investigate whether FA profiles of NEFA may act as a surrogate for AT in 204 male and female participants of varying age and BMI. Furthermore, the profiles are investigated before and after a 12-month period of supplementation with marine $n$-3 PUFA designed to reflect 0-4 portions of oily fish/week and which was demonstrated to modify the FA composition of both plasma NEFA and $\mathrm{AT}^{(13)}$.

\section{Methods}

Data for this analysis were obtained from a two-centre study examining change in FA profiles of nine blood and tissue fractions in response to 12 months of fish oil capsule supplementation equivalent to the amounts of marine $n$ - 3 PUFA provided by $0,1,2$ and 4 portions of oily fish/week ( 1 portion $=1.5 \mathrm{~g}$ EPA and $1.77 \mathrm{~g}$ DHA), with placebo capsules (high oleic acid sunflower oil) taken on the remaining days ${ }^{(13)}$. The study was registered at www.controlled-trials.com as ISRCTN48398526 and is described in detail elsewhere ${ }^{(13)}$. All procedures were approved by the Suffolk Local Research Ethics Committee (approval 05/Q0102/181), and written informed consent was obtained from all participants. The study participants were stratified by age and sex ${ }^{(14)}$, had a BMI range of 18.5-34.9 (median $=25 \cdot 2) \mathrm{kg} / \mathrm{m}^{2}$ and were all described as healthy. The FA pools studied included AT and plasma NEFA, both of which responded in a dose-dependent manner to the EPA and DHA supplementation, such that at 12 months in NEFA the mean observed values for combined EPA+DHA were 0.53 (SD 0.07)\% $(P<0.0001)$ higher per portion (dose), with the 0 portions mean $=1.46$ (sD 0.65). In AT, the values for combined EPA + DHA were 0.05 (SD 0.01$) \%(P<0.0001)$ higher per portion, with the 0 portions mean $=0.40(\mathrm{SD} 0 \cdot 21)$, as previously reported ${ }^{(13)}$.

To monitor background diet, participants completed three unweighed 4-d diet diaries recording food and drink as estimated portions over 3 weekdays and 1 weekend day at 0,6 and 12 months of the intervention period. Data were analysed using an in-house database ${ }^{(15)}$. In addition, during the nine study visits over the 12-month period, participants were asked to report any changes in diet, and were asked specific questions related to cooking oils and spreads and to any white fish consumption, and as previously reported there was no difference in reported dietary FA intake during the 12-month period ${ }^{(13)}$. Fasting blood samples and an abdominal subcutaneous AT biopsy were taken at 0,6 and 12 months during a clinic visit. Plasma NEFA and AT were analysed for FA composition. The preparation and analysis of blood and AT samples has been described previously ${ }^{(13)}$. Briefly, FA were analysed by GC, performed on a Hewlett Packard 6890 gas chromatograph (Hewlett Packard) fitted with a BPX-70 column $(30 \mathrm{~m} \times$ $0.22 \mathrm{~mm} \times 0.25 \mu \mathrm{m}$; Agilent Technologies). The instrument was controlled by, and data were collected using, HPChemStation (Hewlett Packard). FA methyl esters were identified by comparison of retention times with those of authentic standards run previously.

To determine whether NEFA may be used as a biomarker for AT, the agreement between NEFA and AT for individual FA and FA classes (SFA, MUFA, PUFA, $n-3$ PUFA and $n-6$ PUFA) was assessed using Lin's concordance correlation coefficients ${ }^{(16)}$ at the three time points over the 12-month study (0, 6 and 12 months). The relationship between AT and NEFA was also assessed by Spearman's rank correlation coefficients at 0, 6 and 12 months. To assess whether NEFA values are a useful surrogate for AT, linear regression models were fitted to the baseline data from a randomly selected sample ( $70 \%$ of the total cohort, the training set). The outcome variables were FA class in AT with NEFA as a predictor variable. In addition, age, sex and BMI were included as predictor variables where this improved the fit of the models (assessed using $R^{2}$ as a measure of explained variance). These models were then used to predict the FA in AT, using baseline data from the remaining $30 \%$ of the data set (the validation set). The differences between the predicted and observed AT FA values were assessed visually using scatter plots and by the mean absolute percentage error (MAPE). The performance of the predicted values was assessed by splitting the observed and predicted values into tertiles to determine whether the predicted values could be correctly assigned into the low-, medium- or high-FA categories.

All analyses were performed with Stata version 13 (StataCorp LP).

\section{Results}

\section{Study population}

There were 204 participants enrolled into the 12-month study, and 163 participants completed the 12-month visit. The number of participants for whom AT samples were available for comparison with NEFA at each time point and the characteristics of those participants are shown in Table 1.

There were no differences in any of the characteristics or the dietary fat intake of the participants included at each time point.

The baseline participant characteristics for the training and validation sets used to build and test the prediction models are shown in Table 2 . There were no differences in baseline characteristics between the two sets.

\section{Fatty acid profiles in NEFA and adipose tissue in the study} population at baseline

The contributions of SFA and $n-3$ PUFA to the total FA were higher in NEFA than in AT, whereas the contribution of MUFA was lower in NEFA than in AT (Fig. 1). The proportions of $n-6$ PUFA were comparable between NEFA and AT. The five most prevalent FA (oleic, palmitic, linoleic, palmitoleic and stearic acid) were consistent in both pools and accounted for $90.8 \%$ of the FA in AT and $85.6 \%$ of the FA in NEFA (Table 3). DHA, EPA and arachidonic acid accounted for $4 \%$ of NEFA, but only $0.7 \%$ of FA in AT.

\section{Agreement and relationship between fatty acids in adipose} tissue and NEFA

The concordance between AT and NEFA for twenty-eight FA at three time points over the 12-month period is shown in Table 4. Concordance at all three time points was poor $(<0 \cdot 6)$, but it was highest for linoleic acid (18: 2n-6; 0.34-0.53 depending upon the time point). 
Table 1. Characteristics of participants at the three study time points for which there were data available for adipose tissue (AT) and NEFA comparison (Mean values and standard deviations)

\begin{tabular}{|c|c|c|c|c|c|c|c|}
\hline & \multicolumn{2}{|c|}{0 months } & \multicolumn{2}{|c|}{6 months } & \multicolumn{2}{|c|}{12 months } & \multirow[b]{2}{*}{ Difference $(P)^{\star}$} \\
\hline & Mean & SD & Mean & SD & Mean & SD & \\
\hline$n$ (AT samples) & \multicolumn{2}{|c|}{202} & \multicolumn{2}{|c|}{160} & \multicolumn{2}{|c|}{156} & \\
\hline Sex (male \%) & \multicolumn{2}{|c|}{49} & \multicolumn{2}{|c|}{50} & \multicolumn{2}{|c|}{52} & \\
\hline Age (years) & $48 \cdot 4$ & $16 \cdot 1$ & $50 \cdot 2$ & $15 \cdot 4$ & $50 \cdot 7$ & $15 \cdot 2$ & \\
\hline BMI $\left(\mathrm{kg} / \mathrm{m}^{2}\right)$ & $25 \cdot 6$ & 3.88 & $25 \cdot 8$ & 3.85 & $25 \cdot 7$ & 3.92 & 0.30 \\
\hline Body fat (\%) & 21.9 & 8.55 & $22 \cdot 2$ & 7.92 & $21 \cdot 8$ & $8 \cdot 10$ & 0.64 \\
\hline Dietary fat (\%E) & $34 \cdot 1$ & $5 \cdot 76$ & $34 \cdot 1$ & $6 \cdot 17$ & $34 \cdot 1$ & $5 \cdot 37$ & 0.79 \\
\hline Dietary SFA (\%E) & $12 \cdot 8$ & $3 \cdot 12$ & $13 \cdot 1$ & $3 \cdot 30$ & $12 \cdot 7$ & $2 \cdot 83$ & 0.18 \\
\hline Dietary MUFA (\%E) & 11.4 & $2 \cdot 24$ & $11 \cdot 32$ & 2.45 & 11.4 & $2 \cdot 22$ & 0.99 \\
\hline Dietary $n$-3 PUFA (\%E)† & 0.75 & 0.25 & 0.76 & 0.23 & $0 \cdot 76$ & 0.24 & 0.60 \\
\hline Dietary n-6 PUFA (\%E) & $5 \cdot 20$ & 1.76 & 4.90 & 1.35 & $5 \cdot 15$ & 1.65 & 0.17 \\
\hline
\end{tabular}

$\% \mathrm{E}$, percentage of total energy intake.

* The $P$ value of the difference in means between the three time points assessed by repeated-measures ANOVA.

$\dagger$ Excluding $n-3$ PUFA provided as the intervention supplements.

Table 2. Characteristics of participants at baseline in the training set and validation set (Mean values and standard deviations)

\begin{tabular}{|c|c|c|c|c|c|}
\hline & \multicolumn{2}{|c|}{ Training set } & \multicolumn{2}{|c|}{ Validation set } & \multirow[b]{2}{*}{ Difference $(P)^{*}$} \\
\hline & Mean & $\mathrm{SD}$ & Mean & $\mathrm{SD}$ & \\
\hline$n$ & \multicolumn{2}{|c|}{143} & \multicolumn{2}{|c|}{61} & \\
\hline$\%$ population & \multicolumn{2}{|c|}{70} & \multicolumn{2}{|c|}{30} & \\
\hline Sex (male \%) & \multicolumn{2}{|c|}{50} & \multicolumn{2}{|c|}{44} & 0.43 \\
\hline Age (years) & $48 \cdot 4$ & $16 \cdot 5$ & $48 \cdot 5$ & $15 \cdot 6$ & 0.98 \\
\hline BMI $\left(\mathrm{kg} / \mathrm{m}^{2}\right)$ & $25 \cdot 4$ & 3.94 & $26 \cdot 0$ & 3.66 & 0.31 \\
\hline Body fat (\%) & $21 \cdot 3$ & $8 \cdot 55$ & $23 \cdot 2$ & $8 \cdot 34$ & 0.15 \\
\hline Dietary fat (\%E) & 34.0 & 5.48 & $34 \cdot 2$ & 6.43 & 0.85 \\
\hline Dietary SFA (\%E) & $12 \cdot 8$ & $3 \cdot 16$ & $12 \cdot 9$ & $3 \cdot 12$ & 0.80 \\
\hline Dietary MUFA (\%E) & 11.4 & 2.07 & $11 \cdot 3$ & $2 \cdot 25$ & 0.74 \\
\hline Dietary n-6 PUFA (\%E) & 5.09 & $1 \cdot 71$ & $5 \cdot 41$ & $1 \cdot 84$ & 0.24 \\
\hline Dietary n-3 PUFA (\%E)† & 0.75 & 0.23 & 0.74 & 0.31 & 0.70 \\
\hline SFA in AT & $31 \cdot 0$ & $3 \cdot 76$ & $30 \cdot 4$ & $2 \cdot 75$ & 0.27 \\
\hline MUFA in AT & $52 \cdot 7$ & 3.44 & $52 \cdot 7$ & 3.08 & 0.93 \\
\hline$n-6$ PUFA in AT & $13 \cdot 7$ & $2 \cdot 53$ & $13 \cdot 8$ & $2 \cdot 73$ & 0.15 \\
\hline$n$-3 PUFA in AT & 1.64 & 0.32 & 1.61 & 0.28 & 0.52 \\
\hline SFA in NEFA & $36 \cdot 3$ & $3 \cdot 11$ & $37 \cdot 1$ & $4 \cdot 26$ & 0.15 \\
\hline MUFA in NEFA & $42 \cdot 5$ & $5 \cdot 36$ & $42 \cdot 4$ & 4.41 & 0.91 \\
\hline$n-6$ PUFA in NEFA & $13 \cdot 6$ & $2 \cdot 67$ & $13 \cdot 8$ & 1.94 & 0.64 \\
\hline$n$-3 PUFA in NEFA & 4.01 & 1.77 & $3 \cdot 61$ & $1 \cdot 18$ & 0.11 \\
\hline
\end{tabular}

$\% \mathrm{E}$, percentage of total energy intake; AT, adipose tissue.

* Differences $(P)$ between the training and validation set were assessed by $t$ test.

$\dagger$ Excluding $n-3$ PUFA provided as the intervention supplements.

Concordance for total SFA, MUFA and $n-3$ PUFA was poor $(<0 \cdot 1)$ (Table 4). For total $n-6$ PUFA, concordance was $0 \cdot 28$ 0.43 over the course of the 12-month period.

The relationship between AT and NEFA assessed by correlation coefficient was also poor $(<0.59)$ (Table 4$)$. The relationship was strongest between $n-6$ PUFA, but in contrast to the agreement SFA and MUFA were moderately correlated (approximately $0 \cdot 2$ ) at 0 and 6 months (Table 4).

\section{Can NEFA values predict adipose tissue fatty acid values at baseline?}

To test whether NEFA may be used as a surrogate for AT FA profiles, prediction models were fitted to baseline data from the training set ( $70 \%$ of the study population) and then used to predict FA levels in AT in the validation set (the remaining $30 \%$ of the study population). These predicted values were compared with observed values in the validation set (Fig. 2(a) and (b)). We examined n-6 PUFA, which showed moderate concordance between AT and NEFA, and SFA, which showed poor concordance (Table 3).

There was an association for $n-6$ PUFA between NEFA and AT in the training set $(P<0 \cdot 0001)$, although with sex included in the model only $32 \%$ of the variance in the data was explained. Although this model predicted AT $n-6$ PUFA values with a moderate level of error (MAPE of $11 \%$, the predicted values performed poorly at discriminating high and low observed levels of AT $n$ - 6 PUFA (Fig. 2(a)). Predicted values correctly classified $25 \%$ of values of $n-6$ PUFA into low, medium or high categories, but misclassified $75 \%$.

There was a weak association for SFA between NEFA and AT in the training set $(P=0.04)$, which explained only $7 \%$ 
(a)

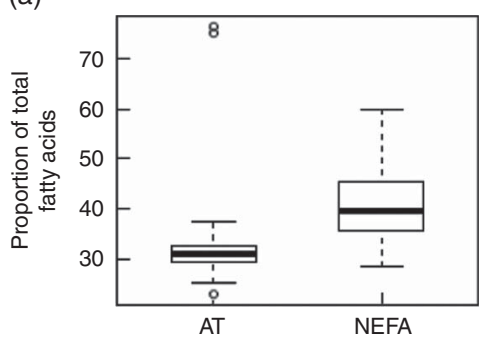

(c)

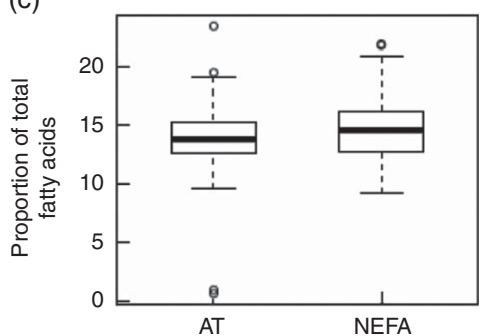

(b)

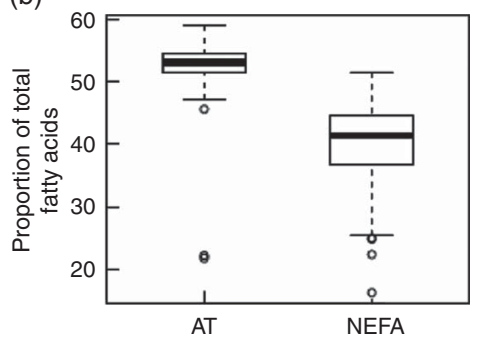

(d)

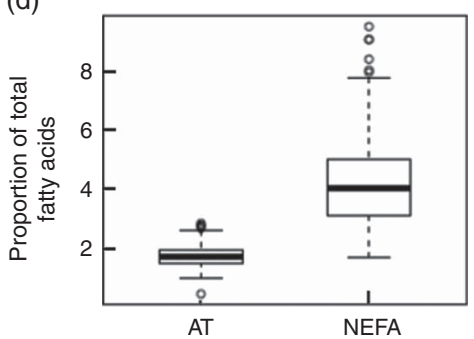

Fig. 1. Comparison of fatty acids by class in adipose tissue (AT) and NEFA at baseline: (a) SFA, (b) MUFA, (c) $n-6$ PUFA, (d) $n-3$ PUFA. Data are median, 25 and 75 th percentile and range for proportion of total fatty acids contributed by each class.

Table 3. Proportion of total of the five most abundant fatty acids (FA) in both adipose tissue (AT) and NEFA fractions and the cumulative proportion of total of these FA at baseline

(Mean values and standard deviations)

\begin{tabular}{|c|c|c|c|c|}
\hline & \multicolumn{2}{|c|}{ AT ( $\%$ of total FA) } & \multicolumn{2}{|c|}{ NEFA ( $\%$ of total FA) } \\
\hline & Mean & SD & Mean & SD \\
\hline Oleic acid (18:1n-9) & $45 \cdot 8$ & $2 \cdot 63$ & $35 \cdot 5$ & $4 \cdot 36$ \\
\hline Palmitic acid (16:0) & $22 \cdot 5$ & 2.48 & $22 \cdot 7$ & $2 \cdot 28$ \\
\hline Linoleic acid (18:2n-6) & $13 \cdot 4$ & 2.72 & $12 \cdot 6$ & 2.49 \\
\hline Palmitoleic acid (16:1n-7) & $5 \cdot 18$ & 1.49 & 3.15 & 1.21 \\
\hline Stearic acid $(18: 0)$ & 3.94 & 1.09 & 11.6 & 2.40 \\
\hline Cumulative total & $90 \cdot 7$ & 1.02 & $85 \cdot 7$ & 3.35 \\
\hline
\end{tabular}

of the variance in the data (including age, sex and BMI in the model). Consequently, although the values for AT SFA predicted from the model in the validation set had moderately low error compared with the observed AT SFA (MAPE of $8 \%$ ), they were poor at discriminating high and low observed levels of SFA (Fig. 2(b)). The predicted values correctly classified $33 \%$ of values into low, medium or high categories of AT SFA according to the observed values, but misclassified $67 \%$.

\section{Can NEFA predict adipose tissue EPA and DHA after an} intervention to increase intake?

There was a strong association between AT and NEFA EPA in the training set at baseline $(P=0.001)$, accounting for age, sex and BMI. However, there was no association following the intervention $(P=0 \cdot 8)$. Similarly, there was an association between AT and NEFA DHA at baseline in the training set $(P=0 \cdot 014)$, but not following the intervention $(P=0 \cdot 3)$. Consequently, these models could not be used to predict proportions of EPA and DHA in AT in the validation set following the intervention.

\section{Discussion}

This study shows that despite the majority of plasma NEFA being derived from AT lipolysis, there was poor agreement between FA in AT and NEFA, particularly for SFA. Furthermore, NEFA cannot be used as a minimally invasive surrogate marker to reflect changes in AT in response to a 12-month intervention to increase EPA and DHA consumption.

Although we, and others ${ }^{(1,5,12)}$, have shown moderate to good correlations between FA in AT and NEFA, the agreement described by the concordance in this study was generally very poor. Previous studies tested the relationship between NEFA and AT by correlation coefficient, which indicated a strong linear relationship between the two values, whereas in our study, as we were interested in whether NEFA values may act as a surrogate marker, we have tested agreement by concordance. In line with the findings in this study, a previous study indicated strong correlations between AT FA and NEFA, but with deviation from 
Table 4. Concordance correlation coefficients between percentage of total fatty acids in adipose tissue and NEFA for each participant at baseline, 6 and 12 months*

\begin{tabular}{|c|c|c|c|c|c|c|}
\hline \multirow[b]{2}{*}{ Fatty acid } & \multicolumn{2}{|c|}{ n 201} & \multicolumn{2}{|c|}{ n 157} & \multicolumn{2}{|c|}{$n 156$} \\
\hline & $\begin{array}{l}\text { Agreement } \\
(0 \text { months })\end{array}$ & $\begin{array}{l}\text { Correlation } \\
\text { (0 months) }\end{array}$ & $\begin{array}{l}\text { Agreement } \\
(6 \text { months })\end{array}$ & $\begin{array}{l}\text { Correlation } \\
\text { (6 months) }\end{array}$ & $\begin{array}{c}\text { Agreement } \\
\text { (12 months) }\end{array}$ & $\begin{array}{c}\text { Correlation } \\
\text { (12 months) }\end{array}$ \\
\hline $10: 0$ & -0.01 & -0.02 & 0.08 & 0.06 & 0.02 & 0.14 \\
\hline $12: 0$ & -0.01 & -0.15 & 0.01 & 0.04 & -0.09 & -0.09 \\
\hline $13: 0$ & -0.09 & -0.07 & -0.05 & -0.04 & -0.0 & -0.07 \\
\hline $14: 0$ & 0.02 & 0.13 & 0.05 & 0.25 & 0 & 0.10 \\
\hline $14: 1 n-9$ & 0.06 & 0.26 & 0.01 & 0.15 & 0.41 & 0.59 \\
\hline $15: 0$ & 0.14 & 0.22 & 0.36 & 0.42 & -0.01 & 0.15 \\
\hline $15: 1$ & 0.01 & 0.06 & -0.08 & -0.02 & -0.05 & -0.01 \\
\hline $16: 0$ & 0.21 & 0.31 & 0.28 & 0.35 & 0.06 & 0.20 \\
\hline $16: 1 n-7$ & 0.16 & 0.29 & 0.26 & 0.55 & 0.17 & 0.45 \\
\hline $17: 0$ & 0.01 & 0.12 & 0.01 & 0.24 & -0.03 & -0.06 \\
\hline $17: 1 n-8$ & -0.15 & -0.30 & -0.03 & 0.01 & 0.33 & 0.32 \\
\hline $18: 0$ & 0.01 & 0.20 & 0.01 & 0.28 & -0.01 & -0.06 \\
\hline $18: 1 n-9$ & 0.01 & 0.16 & 0.02 & 0.22 & -0.02 & -0.08 \\
\hline $18: 2 n-6$ & 0.42 & 0.43 & 0.53 & 0.57 & 0.34 & 0.47 \\
\hline $18: 3 n-6$ & 0.02 & 0.14 & 0.09 & 0.23 & -0.01 & 0.00 \\
\hline $18: 3 n-3$ & 0.21 & 0.20 & 0.39 & 0.51 & 0.22 & 0.35 \\
\hline $20: 0$ & 0.11 & 0.10 & 0 & -0.04 & 0.06 & 0.27 \\
\hline $20: 1 n-9$ & 0 & 0.07 & 0.06 & 0.29 & 0.34 & 0.33 \\
\hline $20: 2 n-6$ & 0.03 & 0.25 & 0.18 & 0.17 & 0.02 & 0.04 \\
\hline $20: 3 n-3$ & -0.04 & -0.11 & 0.05 & 0.16 & 0 & 0.03 \\
\hline $20: 3 n-6$ & 0.01 & 0.34 & 0 & 0.03 & 0.08 & 0.26 \\
\hline $20: 4 n-6$ & 0 & 0.04 & 0.01 & 0.07 & -0.01 & -0.08 \\
\hline $20: 5 n-3$ & 0.06 & 0.27 & 0.01 & 0.13 & 0.03 & 0.21 \\
\hline $22: 0$ & 0.03 & -0.01 & -0.01 & -0.08 & 0.05 & 0.02 \\
\hline $22: 4 n-6$ & 0.02 & 0.06 & 0.03 & 0.03 & 0.08 & 0.18 \\
\hline $22: 5 n-3$ & 0.01 & 0.04 & -0.04 & -0.06 & -0.01 & -0.01 \\
\hline $22: 5 n-6$ & 0.01 & 0.02 & 0 & 0.03 & 0.02 & 0.01 \\
\hline $22: 6 n-3$ & 0.02 & 0.24 & 0.01 & 0.16 & 0.01 & 0.21 \\
\hline SFA & 0.07 & 0.22 & 0.05 & 0.28 & -0.06 & -0.09 \\
\hline MUFA & 0.03 & 0.17 & 0.03 & 0.24 & -0.01 & 0.01 \\
\hline$n-6$ PUFA & 0.28 & 0.51 & 0.43 & 0.59 & 0.34 & 0.47 \\
\hline$n-3$ PUFA & 0.01 & 0.16 & 0.01 & 0.17 & 0.02 & 0.16 \\
\hline
\end{tabular}

* Concordance assessed by Lin's correlation coefficient. Agreement assessed by Spearman's correlation coefficient.

(a)

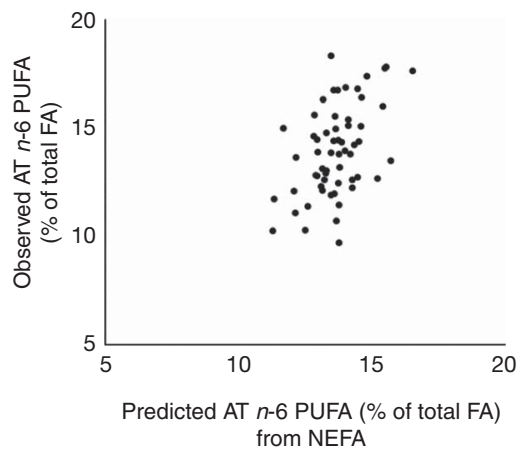

(b)

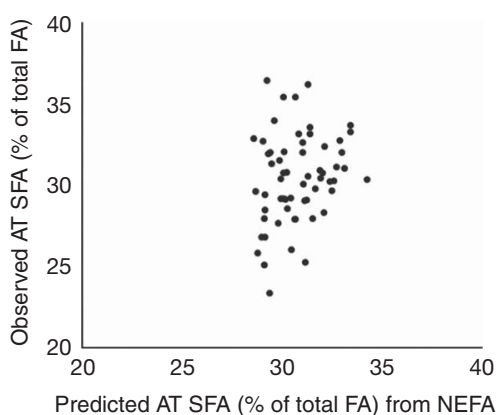

Fig. 2. Predicted adipose tissue (AT) $n-6$ PUFA and SFA compared with observed AT values at baseline. Predicted AT $n$ - 6 PUFA values (a) and SFA values (b) were generated from equations of comparison of NEFA $n-6$ PUFA and SFA with AT and including age, sex and BMI in the training set. These predicted values for AT $n-6$ PUFA and SFA were compared with the observed values in the validation set. FA, fatty acids.

the line of agreement (the identity line), particularly for $n$-3 PUFA and SFA $(16: 0 \text { and } 18: 0)^{(12)}$. In our study, the poor concordance was reflected by a poor ability to predict high and low AT FA values, particularly for SFA (Fig. 2(b)).

The agreement between AT and NEFA was markedly better for $n-6$ PUFA than any other FA class, as has been found previously in a cohort of patients with myocardial infarction and age-matched controls ${ }^{(12)}$ and in a cohort of women who were predominantly obese ${ }^{(5)}$. FA are selectively mobilised according to structure ${ }^{(17)}$; generally, mobilisation is greater for essential $\mathrm{FA}^{(18)}$, greater with the degree of saturation, but lower with chain length for any degree of saturation ${ }^{(19)}$. This may explain 
the greater prevalence of EPA, DHA and arachidonic acid seen in NEFA compared with AT. It has also been suggested that other factors such as physical activity ${ }^{(6,20)}$, BMI ${ }^{(5)}$ and insulin sensitivity $^{(21)}$ contribute to selective mobilisation of FA. In addition, differences between AT FA profile and NEFA are also contributed to by de novo lipogenesis in particular contributions of SFA, preferential postprandial uptake of $\mathrm{FA}^{(4)}$ and utilisation of $n-6$ and n-3 PUFA such as arachidonic acid, EPA and DHA for synthesis of eicosanoids and other bioactive compounds ${ }^{(22)}$.

We explored further whether NEFA may be used as minimally invasive markers of changes in AT FA composition in response to changes in $n$-3 PUFA intake. In this study, we demonstrated that there was poor concordance between EPA (20:5n-3) and DHA (22: 6n-3) before and in response to an intervention to increase EPA and DHA intake. There was an association between AT and NEFA for both EPA and DHA before, but not after, the intervention. This indicates that the supplementation resulted in EPA and DHA changes in one pool that were not mirrored in the other pool. Because of the lack of association, we were unable to create prediction equations to test the accuracy of NEFA as a biomarker of AT $n-3$ PUFA, as we had done for $n$ - 6 PUFA and SFA. However, it is clear from the very poor concordance and the lack of association that NEFA EPA and DHA would not be able to predict AT EPA and DHA with any accuracy. Although EPA and DHA in both AT and NEFA are increased in accordance with an increase in intake $^{(13,23,24)}$, there is only a moderate correlation between change in AT and NEFA EPA + DHA (Spearman's correlation= 0.27), and we have demonstrated here that changes in AT cannot be predicted from changes in NEFA. We have suggested previously that blood markers, particularly mononuclear cells, may be better at discriminating long-term changes in EPA and DHA intake than $\mathrm{AT}^{(13)}$

AT FA profile has important implications for biological function; in particular, proportions of arachidonic acid, EPA and DHA affect the inflammatory profile ${ }^{(22,25)}$. Although there are strong correlations between AT FA in subcutaneous and visceral depots, differences in proportions of specific FA between the depots have been found; for example, FA of longer chain length (>22 C) are more predominant in subcutaneous AT and highly unsaturated FA in visceral depots ${ }^{(5)}$. In addition, there are differences in FA profile and metabolism between subcutaneous AT depots of different origins ${ }^{(26,27)}$. This may contribute to the lack of concordance found between AT and NEFA in this study, and it would limit the utility of NEFA as a surrogate for AT FA, as profile differences important for AT biological function would not be reflected in the overall NEFA profile.

This study has several strengths, as it assessed the agreement of a number of FA (twenty-eight) between AT and NEFA at repeated time points over a 12 -month period. We measured forty FA, but because of low amounts in one or other of the pools data for twelve FA could not be used. This study was conducted in male and female participants of a wide age and BMI range, representative of the population, and in a comparatively large number of individuals. We also assessed the utility of NEFA as a surrogate for changes in AT FA in response to a 12-month intervention to increase EPA and DHA consumption. A limitation of this study is that AT was only sampled from the abdominal subcutaneous depot. There are differences in FA profile and metabolism of FA between subcutaneous and visceral depots, as well as between subcutaneous depots at different locations ${ }^{(26,28)}$, which may explain the poor agreement between NEFA and AT FA found in the current study. It is possible the NEFA better reflect the FA profile of visceral AT, and as this depot is even more invasive to sample from it would be useful to know whether NEFA are a good reflection of this more biologically active depot ${ }^{(28)}$.

In conclusion, despite moderate correlation between AT and NEFA FA profiles, NEFA are not a suitable surrogate for AT FA.

\section{Acknowledgements}

The authors acknowledge the contributions of Joanna Gambell, Louise Timbers, Clionadh O'Reilly, Mariana Eberhard, Katey Bergstralh, Sarah Gibbings (Research Assistants), Darren Cole (Database Manager), Laura Wang, Stephen Young, Sadiq Lula, Christine Clewes, Christiaan Gelauf, Jade Pretorius (fatty acid analysis), Alison Lennox, Birgit Teucher, Anna Gent, Celia Greenberg (coding and analysis of dietary data), Mario Siervo, Rosemary Hall and Sue Fisher (Clinical Support). We appreciate the contribution to the original trial of Adrian Mander, as well as statistical advice for this study. We thank Leanne Hodson for useful discussions.

The UK Medical Research Council supported L. M. B., C. G. W., S. A. J. (grant code U1059.60.389) and L. S. (grant code U1052.00.014) for this work. The original trial was funded by the UK Foods Standards Agency (N05065/66). The views expressed are those of the authors and do not necessarily reflect UK Government policy or advice. Equazen (Wallingford, Oxford, UK) supplied the capsules for the intervention.

C. G. W. and P. C. C. conceived this study; C. G. W. and L. S. conducted the analysis; C. G. W. drafted the manuscript; L. M. B., S. A. J. and P. C. C. designed the original trial; L. M. B., C. G. W., A. L. W. and J. M. conducted the research on the original trial. All authors critically appraised the manuscript.

The authors declare no conflicts of interest relevant to this paper.

\section{References}

1. Hodson L, Skeaff C \& Fielding B (2008) Fatty acid composition of adipose tissue and blood in humans and its use as a biomarker of dietary intake. Prog Lipid Res 47, 348-380.

2. Brevik A, Veierod MB, Drevon CA, et al. (2005) Evaluation of the odd fatty acids 15:0 and 17:0 in serum and adipose tissue as markers of intake of milk and dairy fat. Eur J Clin Nutr 59 , 1417-1422.

3. Baylin A, Kabagambe EK, Siles X, et al. (2002) Adipose tissue biomarkers of fatty acid intake. Am J Clin Nutr 76, 750-757.

4. Summers LK, Barnes SC, Fielding BA, et al. (2000) Uptake of individual fatty acids into adipose tissue in relation to their presence in the diet. Am J Clin Nutr 71, 1470-1477.

5. Hellmuth C, Demmelmair H, Schmitt I, et al. (2013) Association between plasma nonesterified fatty acids species and adipose tissue fatty acid composition. PLOS ONE 8, e74927. 
6. Sutherland WHF, Woodhouse SP \& Heyworth MR (1981) Physical training and adipose tissue fatty acid composition in men. Metabolism 30, 839-844.

7. Iggman D, Ärnlöv J, Vessby B, et al. (2010) Adipose tissue fatty acids and insulin sensitivity in elderly men. Diabetologia 53, 850-857.

8. Kratz M, Kuzma JN, Hagman DK, et al. (2013) n3 PUFAs do not affect adipose tissue inflammation in overweight to moderately obese men and women. J Nutr 143, 1340-1347.

9. Bulló M, Casas-Agustench P, Amigó-Correig P, et al. (2007 Inflammation, obesity and comorbidities: the role of diet. Public Health Nutr 10, 1164-1172.

10. Siriwardhana N, Kalupahana NS, Cekanova M, et al. (2013) Modulation of adipose tissue inflammation by bioactive food compounds. J Nutr Biochem 24, 613-623.

11. Frayn KN \& Humphreys SM (2012) Metabolic characteristics of human subcutaneous abdominal adipose tissue after overnight fast. Am J Physiol Endocrinol Metab 302, E468-E475.

12. Yli-Jama P, Haugen TS, Rebnord HM, et al. (2001) Selective mobilisation of fatty acids from human adipose tissue. Eur J Intern Med 12, 107-115.

13. Browning LM, Walker CG, Mander AP, et al. (2012) Incorporation of eicosapentaenoic and docosahexaenoic acids into lipid pools when given as supplements providing doses equivalent to typical intakes of oily fish. Am J Clin Nutr 96 748-758.

14. Walker CG, Browning LM, Mander AP, et al. (2014) Age and sex differences in the incorporation of EPA and DHA into plasma fractions, cells and adipose tissue in humans. Br J Nutr 111, 679-689.

15. Fitt E, Cole D, Ziauddeen N, et al. (2014) DINO (Diet In Nutrients Out) - an integrated dietary assessment system. Public Health Nutr 18, 234-241.

16. Lin L (1989) A concordance correlation coefficient to evaluate reproducibility. Biometrics 45, 255-268.
17. Raclot T, Holm C \& Langin D (2001) Fatty acid specificity of hormone-sensitive lipase: implication in the selective hydrolysis of triacylglycerols. J Lipid Res 42, 2049-2057.

18. Raclot T, Langin D, Lafontan M, et al. (1997) Selective release of human adipocyte fatty acids according to molecular structure. Biochem J 15, 911-915.

19. Halliwell KJ, Fielding BA, Samra JS, et al. (1996) Release of individual fatty acids from human adipose tissue in vivo after an overnight fast. J Lipid Res 37, 1842-1848.

20. Mougios V, Kotzamanidis C, Koutsari C, et al. (1995) Exerciseinduced changes in the concentration of individual fatty acids and triacylglycerols of human plasma. Metabolism 44, 681-688.

21. Yue KTN, Phillips PE, Davis JW, et al. (1981) Effect of glucose on plasma concentrations of individual non-esterified fatty acids of non-diabetic and insulin-independent diabetic men. Postgrad Med J 57, 622-626.

22. Calder PC (2006) n-3 Polyunsaturated fatty acids, inflammation, and inflammatory diseases. Am J Clin Nutr 83, 1505S-1519S.

23. Leaf DA, Connor WE, Barstad L, et al. (1995) Incorporation of dietary $n-3$ fatty acids into the fatty acids of human adipose tissue and plasma lipid classes. Am J Clin Nutr 62, 68-73.

24. Wall R, Ross RP, Fitzgerald GF, et al. (2010 Fatty acids from fish: the anti-inflammatory potential of long-chain omega-3 fatty acids. Nutr Rev 68, 280-289.

25. Fan C, Zirpoli H \& Qi K (2013) n-3 Fatty acids modulate adipose tissue inflammation and oxidative stress. Curr Opin Clin Nutr Metab Care 16, 124-132.

26. Malcom GT, Bhattacharyya AK, Velez-Duran M, et al. (1989) Fatty acid composition of adipose tissue in humans: differences between subcutaneous sites. Am J Clin Nutr 50, 288-291.

27. Pinnick KE, Neville MJ, Fielding BA, et al. (2012) Gluteofemoral adipose tissue plays a major role in production of the lipokine palmitoleate in humans. Diabetes 61, 1399-1403.

28. Ibrahim MM (2010) Subcutaneous and visceral adipose tissue: structural and functional differences. Obes Rev 11, 11-18. 